\title{
Heat Treatment of Chinese Quince Polyphenols Increases Rat Plasma Levels of Protocatechuic and Vanillic acids
}

Yasunori Hamauzu*, Naotaka Takedachi, Rumika Miyasaka and Hidefumi Makabe

Department of Sciences of Functional Foods, Graduate School of Agriculture, Shinshu University, 8304 Minamiminowa 399-4598, Japan

* Author to whom correspondence should be addressed.

Tel +81-265-77-1413; Fax +81-265-77-1700; E-mail hamauzu@ shinshu-u.ac.jp 


\begin{abstract}
The effect of heat-treated Chinese quince polyphenols on plasma antioxidant activity and the plasma levels of aromatic acids after oral administration to rats was investigated. The aqueous solution of Chinese quince polyphenols $(\mathrm{CP})$ that had been extracted using $60 \%(\mathrm{v} / \mathrm{v})$ acetone turned reddish during the heat treatment in the presence of citric acid. During the treatment, decreases in polymeric procyanidins and an increase in monomers, dimers and oligomers were observed. In the oral administration study, CP subjected to $2 \mathrm{~h}$ of heat treatment (CPT-2) caused a significant increase in antioxidant activity as assessed by the ferric reducing ability of plasma (FRAP) method. In addition, $\mathrm{CP}, \mathrm{CP}+$ citric acid and $\mathrm{CP}$ subjected to $8 \mathrm{~h}$ of heat treatment (CPT-8) also tended to increase the FRAP value but the increase was not significant. Of the plasma aromatic acids detected, protocatechuic acid increased in all rats given the polyphenols. Vanillic acid increased significantly in rats given CPT-2 and CPT-8, reflecting a facilitatory effect of heat treatment on the intestinal absorption of protocatechuic acid and subsequent conversion by catechol- $O$-methyl transferase. These results suggested that the heat-induced breakdown of Chinese quince polyphenols seems to be an effective method of leading to an elevation of plasma antioxidant activity and levels of aromatic acid that are health-benefiting compounds derived from procyanidins.
\end{abstract}

Keywords: phenolics; flavan-3-ol; procyanidins; heating; breakdown; antioxidant activity; FRAP; aromatic acid; metabolism 


\section{INTRODUCTION}

The Chinese quince (Pseudocydonia sinensis Schneider) is native to China, and was imported to Japan in early times (apparently around the Heian period). The fruit has woody hard flesh, strong acidity and astringency, rendering the raw fruit inedible. However, Chinese quinces have been consumed in the form of fruit liquor, jam, and glutinous starch syrup, etc., much as the fruit of normal quinces have. Although the flesh is harder than that of normal quince fruit, Chinese quince fruit could be used to produce quince jelly (such as Spanish 'membrillo', Portuguese 'marmelada' or French 'cotignac'). Products made from Chinese quinces normally have higher concentrations of polyphenols and greater antioxidant properties than those made from normal quinces because Chinese quince fruit has almost four times the polyphenol content of quince fruit (Hamauzu, Yasui, Inno, Kume \& Omanyuda, 2005).

When Chinese quince fruit is cooked in the production of jam (glutinous starch syrup) or decocted syrup, it is sometimes subjected to long-term heating, especially when the products need to have a bright red color. Although the polyphenols of Chinese quince fruit have been reported to have various properties such as in vitro antioxidative, antiinfluenza viral (Hamauzu et al., 2005), anti-inflammatory (Osawa, Miyazaki, Imai, Arakawa, Yasuda \& Takeya, 1999), antiulcerative (Hamauzu, Inno, Kume, Irie \& Hiramatsu, 2006) and antipruritic (Oku, Ueda \& Ishiguro, 2003) activities, there have been no reports on the effect of heating during processing on polyphenolic composition and biological activity. We previously reported that Chinese quince fruit is rich in procyanidins, which contribute, during heating, to the reddish coloration of products made from it (Hamauzu, Kume, Yasui \& Fujita, 2007). Moreover, the in vitro antioxidant activity of an aqueous solution of semi-purified polyphenols increased when heated with citric acid for up to $12 \mathrm{~h}$. This raises the question of whether heat treatment also increases their antioxidant properties in vivo after oral administration to rats. Since 
procyanidins, even oligomers, are unlikely to be absorbed in their intact forms and are reported to be absorbed after being metabolized to various aromatic acids by intestinal microflora, we measured the plasma concentration of aromatic acids as an indicator of the effect of procyanidin intake. Moreover, some aromatic acids are reported to have health benefits and, essentially, are one active form of procyanidins in vivo.

In this study, the breakdown of Chinese quince polyphenols during long-term heat treatment and the effect of the treatment on their antioxidant activity (ferric reducing ability) and on the concentration of aromatic acid metabolites in the plasma of rats after oral administration were investigated.

\section{MATERIALS AND METHODS}

\subsection{Plant Materials}

The ripe fruits of Chinese quince (Pseudocydonia sinensis Schneider var. Toukarin) were obtained from a local orchard in Nagano Prefecture, Japan.

\subsection{Chemicals and Reagents}

(-)-Epicatechin, vanillic acid, 4-hydroxybenzoic acid, b-glucuronidase type $\mathrm{H}-2$ (including sulfatase), and 2,4,6-tri(2-pyridyl)-s-triazine (TPTZ) were purchased from Sigma-Aldrich, Ltd. (St. Louis, MO, USA). Cyanidin chloride was purchased from Funakoshi Co. (Tokyo, Japan). Salicylic acid, protocatechuic acid, hippuric acid, citric acid were purchased from Nacalai Tesque, Inc. (Kyoto, Japan). 3-Hydroxyphenylacetic acid was purchased from Alfa Aesar (Karlsruhe, Germany). Solvents were purchased from Nacalai Tesque. Folin-Ciocalteu reagent was purchased from Wako Pure Chemical Industries, Ltd. (Osaka, Japan). Procyanidin B2 was purchased from Funakoshi Co., Ltd. (Tokyo, Japan). The other dimers (B1, B3 and B4) were chemically 
synthesized from catechin and epicatechin (Mohri, Sagehashi, Yamada, Hattori, Morimura, Kamo et al., 2007).

\subsection{Extraction of Crude Polyphenols (CP) from Chinese Quince Fruit}

The semi-purified Chinese quince polyphenols were prepared using the procedure described in a previous report (Hamauzu et al, 2007). The freeze-dried powder of the flesh (30 g) was washed with $300 \mathrm{~mL}$ of petroleum ether to remove lipids and then the polyphenols were extracted with $60 \%$ aqueous acetone $(300 \mathrm{~mL}$, three times), rotary evaporated, and semi-purified using a Sep-Pak Vac $20 \mathrm{~cm}^{3}$ (5 g) C18 cartridge (Waters Co., Milford, MA). The end result was approx. $3.0 \mathrm{~g}$ of freeze-dried crude polyphenols (CP) in powder form.

\subsection{Breakdown of CP by Heating with Citric Acid (Preparation of CPT)}

An aqueous solution of $\mathrm{CP}$ (final conc. of $0.1 \%$, w/v, as epicatechin equivalent) was heated in the presence of citric acid (final conc. of $2.0 \%, \mathrm{w} / \mathrm{v}$ ) in a screw-capped test tube for $0,2,4,8$, or $12 \mathrm{~h}$ using a dry bath incubator (GENIUS two block, Nippon Genetics Co., Ltd., Tokyo, Japan) at $95^{\circ} \mathrm{C}$. For the in vivo experiment, CP subjected to the same heat treatment for $0 \mathrm{~h}(\mathrm{CPT}-0=\mathrm{CP}$ plus citric acid $), 2 \mathrm{~h}(\mathrm{CPT}-2)$ and $8 \mathrm{~h}$ (CPT-8) were prepared for administration to the rats.

\subsection{Total Phenolic Assay}

The experimental procedure was adapted from that of Hamauzu et al. (2007). Two $\mathrm{mL}$ of sample solution were mixed with $2 \mathrm{~mL}$ of diluted Folin-Ciocalteu reagent $(1 \mathrm{~N})$ in a test tube. After $3 \mathrm{~min}, 2 \mathrm{~mL}$ of $\mathrm{Na}_{2} \mathrm{CO}_{3}(10 \mathrm{~g} / 100 \mathrm{~mL})$ were added and the mixture was incubated for $60 \mathrm{~min}$ at room temperature. The absorbance was measured at $700 \mathrm{~nm}$ with a Shimadzu UV-1200 spectrophotometer (Tokyo, Japan) against a blank 
( $2 \mathrm{~mL}$ of deionized water, plus reagents) in the reference cell. (-)-Epicatechin was used as the standard $(r=0.99975)$.

\subsection{Reversed Phase HPLC}

Chromatographic separation was carried out on a Luna $5 \mu$ C18 column $(150 \times 4.6$ mm, Phenomenex, Inc., Torrance, CA., USA) with a security guard cartridge $(3.0 \times 4.6$ $\mathrm{mm})$ at $40^{\circ} \mathrm{C}$. Solvents were $0.1 \%$ trifluoroacetic acid (A) and $0.1 \%$ trifluoroacetic acid in acetonitrile (B). The gradient program began with 5\% B and was changed to obtain $15 \% \mathrm{~B}$ at $30 \mathrm{~min}, 32 \% \mathrm{~B}$ at $35 \mathrm{~min}, 40 \% \mathrm{~B}$ at $45 \mathrm{~min}$, and $75 \% \mathrm{~B}$ at $50 \mathrm{~min}$. The $75 \% \mathrm{~B}$ was maintained until $65 \mathrm{~min}$. The flow rate was $1.0 \mathrm{~mL} / \mathrm{min}$ and the injection volume was $20 \mu \mathrm{L}$. Detection was performed at $280 \mathrm{~nm}$ for flavan-3-ols and $325 \mathrm{~nm}$ for hydroxycinnamic derivatives on a Shimadzu SPD-M10Avp photodiode array detector. For the detection of reddish colored substances, $458 \mathrm{~nm}$ and $525 \mathrm{~nm}$ were also used. Peaks were identified by comparing retention times and UV-visible spectra with those of standards. Polymeric and oligomeric procyanidins were identified by comparing the absorption spectra with that of (-)-epicatechin, and the denaturation of them was confirmed by thioacidolysis (Hamauzu et al., 2007) as well as the spectral changes. Presence of protocatechuic and vanillic acids in the Chinese quince polyphenols subjected to heat treatment was also checked by retention time and absorption spectra of peaks on HPLC chromatogram.

\subsection{Animals}

Male Wistar rats weighing 230-270 g were conditioned in standard boxes for 3 days before the experiments. They were fed a standard laboratory diet (CE-2, CLEA Japan, Inc.), provided with water ad libitum and kept on a 12/12 h light-dark cycle. The animals were fasted $24 \mathrm{~h}$ before the experiment but allowed free access to water. Use of 
animals for the experiment was approved by the Ethics Committee of Shinshu University.

\subsection{Oral Administration of Polyphenols and Blood Sampling}

Each rat received (oral administration) approx. $40 \mathrm{mg}$ of $\mathrm{CP}, \mathrm{CPT}$ or $\mathrm{RCP}$ (containing $20 \mathrm{mg}$ of polyphenols as (-)-epicatechin equivalent estimated by the Folin-Ciocalteu method) suspended in $2 \mathrm{~mL}$ of water. Each sample destined for heat treatment (CPT-2 and CPT-8) had its (-)-epicatechin equivalent $(20 \mathrm{mg})$ confirmed before heat treatment because the estimation of phenolic content by the Folin-Ciocalteu method is affected by heating. Control rats were given $2 \mathrm{~mL}$ of water. Two hours after administration, rats were anaesthetized with diethylether, the abdominal wall was opened, and blood was collected from the abdominal aorta into heparinized tubes. The plasma was separated from the blood solids by centrifugation $\left(800 \times g\right.$ for 5 min at $4^{\circ} \mathrm{C}$ ).

\subsection{The FRAP Assay in Rats}

The experimental procedure for the FRAP (ferric reducing ability of plasma) method basically followed that of Benzie \& Strain (1996) with minor modifications. The FRAP method reagent was prepared as a mixture of $10 \mathrm{~mL}$ of $300 \mathrm{mM}$ acetate buffer (pH 3.6), $1 \mathrm{~mL}$ of $10 \mathrm{mM}$ TPTZ-40 mM hydrochloric solution, and $1 \mathrm{~mL}$ of 20 $\mathrm{mM} \mathrm{FeCl} 3$ solution. The FRAP reagent, once prepared, was immediately incubated for $10 \mathrm{~min}$ at $37^{\circ} \mathrm{C}$, then $1.5 \mathrm{~mL}$ of the reagent was added to $50 \mu \mathrm{L}$ of the plasma sample. The reaction mixture was incubated for $4 \mathrm{~min}$ at room temperature and the absorbance at $593 \mathrm{~nm}$ was measured using a UV-1200 spectrophotometer.

\subsection{Analysis of Aromatic Acid Metabolites}


Plasma ( $2 \mathrm{~mL}$ ) was added to $1.05 \mathrm{~mL}$ of $0.58 \mathrm{mM}$ acetic acid to obtain a $\mathrm{pH}$ of 4.9, and $1000 \mathrm{U}$ of b-glucuronidase Type H-2 containing $50 \mathrm{U}$ of sulfatase was added and then incubated at $37^{\circ} \mathrm{C}$ for $45 \mathrm{~min}$. After acidification to $\mathrm{pH} 2$ with $6 \mathrm{M} \mathrm{HCl}(46$ $\mu \mathrm{L})$, the solution was then extracted twice with ethyl acetate $(4 \mathrm{~mL})$ and centrifuged at $2400{ }^{-} g, 4^{\circ} \mathrm{C}$ for $10 \mathrm{~min}$. The resulting supernatant was evaporated under an $\mathrm{N}_{2}$ gas stream and then re-dissolved in $500 \mu \mathrm{L}$ of $25 \%(\mathrm{v} / \mathrm{v})$ methanol. A $200 \mu \mathrm{L}$ sample of the solution was added to $1800 \mu \mathrm{L}$ of $10 \mu \mathrm{M}$ salicylic acid as an internal standard and analyzed by Waters LC-electrospray ionization (ESI)-MS/MS system (Quattro Micro API, Micromass, UK). For the quantitative analysis of aromatic acid metabolites, single ion monitoring (SIR) mode was used. The MS/MS mode was also used for qualitative analysis by monitoring the product ions.

\subsection{LC-ESI-MS Conditions}

The column was a Luna $5 \mu \mathrm{C} 18$ column $(150 \times 4.6 \mathrm{~mm})$ with a security guard cartridge $(3.0 \times 4.6 \mathrm{~mm})$ and the mobile phases consisted of $5 \%$ acetonitrile in $0.1 \%$ aqueous formic acid (solvent $\mathrm{A}$ ) and $50 \%$ acetonitrile in $0.1 \%$ aqueous formic acid (solvent B). The flow rate was $0.3 \mathrm{~mL} / \mathrm{min}$. The following gradient was applied: $0-40$ min, linear gradient from $25 \% \mathrm{~B}$ to $50 \% \mathrm{~B}$; $40-60 \mathrm{~min}$, from $50 \%$ B to $100 \% \mathrm{~B}$; $60-65$

min, $100 \%$ B. Detection was carried out using ESI conducted in negative mode with a capillary voltage of $-3 \mathrm{kV}$. A cone voltage of $-20 \mathrm{~V}$ was applied for the detection of aromatic acids and $-40 \mathrm{~V}$ for catechin metabolites. A source temperature of $120^{\circ} \mathrm{C}$ and a desolvation temperature of $350^{\circ} \mathrm{C}$ were applied. The cone gas and desolvation gas flow were 50 and $600 \mathrm{~L} / \mathrm{h}$, respectively. MS data collected were processed using MassLynx v.4.0.

\subsection{LC-ESI-MS/MS Conditions}


The peaks in the total ion chromatogram (TIC) obtained by MS scan or SIR mode were further confirmed using the same LC-ESI-MS/MS system described above. Argon gas was used as a collision gas, and the potential change defining the collision energy was optimized in the range of $10-20 \mathrm{~V}$ as appropriate for both parent and product ions. Aromatic acid metabolites and salicylic acid (internal standard) were detected according to the respective $m / z$ values of their parent and product ions: 4-hydroxybenzoic acid (137/93), protocatechuic acid (153/109), vanillic acid (167/123), 3-hydroxyphenylacetic acid (151/107), hippuric acid (178/134) and salicylic acid (137/93).

\subsection{Statistics}

Data are expressed as means \pm SE. Means were compared by one-way ANOVAs followed by Tukey-Kramer tests using Excel 2004 with the add-in software Statcel 2 (OMS, Tokyo, Japan). Differences with $P<0.05$ were considered significant.

\section{RESULTS AND DISCUSSION}

\subsection{Changes in Polyphenolic Profile during Heat Treatment}

During $12 \mathrm{~h}$ of the heat treatment, the $\mathrm{CP}$ solution turned reddish, and the color intensity (measured at $458 \mathrm{~nm}$ ) increased linearly as described previously (Hamauzu et al., 2007). During the treatment, increases in (+)-catechin, (-)-epicatechin, procyanidin dimers (B1-B4) and other oligomeric procyanidins were observed (Figures 1 and 2). The increase in monomeric catechins (catechin plus epicatechin) and in dimers was considerable in the first $2 \mathrm{~h}$. Monomers increased such that their concentrations were approx. 4 times the initial concentration after $2 \mathrm{~h}$ of heating, and they reached 5.4 times the initial value at $12 \mathrm{~h}$ after the treatment. (+)-Catechin that was not detected in initial solution rapidly increased during $2 \mathrm{~h}$ of heating and slightly decreased thereafter. Meanwhile, (-)-epicatechin increased continuously over $12 \mathrm{~h}$ of heating and reached 3.8 
times its initial concentration. The pattern of change in total dimers was similar to that of monomers; it increased to be 3 times the initial concentration after the first $2 \mathrm{~h}$ and slightly increased thereafter to be 4 times the initial concentration.

In addition, some compounds having an absorption maximum at $280 \mathrm{~nm}$ (with a spectrum shape similar to that of catechin) appeared as peaks on the RP-HPLC chromatogram at early retention times. Small amount of protocatechuic acid was detected in Chinese quince polyphenols before heating and the amount seemed to increase by a long-term heating because that a clear absorption spectra peculiar to protocatechuic acid was observed in a peak of CPT-12. However, this was unclear in the same peak of CPT-2 and CPT-8 because the peak was not separated from another peak that was appeared by heat treatment. Meanwhile, vanillic acid was not detected in any polyphenolic samples with or without heat treatment. In the chromatogram, a peak of cyanidin and two peaks having an absorption maximum at $458 \mathrm{~nm}$ were observed as well as the previous report (Hamauzu et al., 2007) (data not shown). The unseparated clump of polymeric procyanidins decreased (approx. 30\%) after $2 \mathrm{~h}$ of heating, and increased thereafter (Figure 2). However, the increase was accompanied by the increase of visible absorbency (around 400-550 $\mathrm{nm}$ ) indicating that polymeric procyanidins had been denatured and then re-polymerized to novel polyphenols during heat treatment.

The changes in the in vitro antioxidant properties and metal reducing ability were confirmed and the same trend was observed as that described previously (Hamauzu et al., 2007); they markedly increased during the first $2 \mathrm{~h}$ of heat treatment and the high levels were maintained until the end of the treatment (data not shown).

From the results, Chinese quince polyphenols heated with $2 \%$ citric acid seem to change from having features characteristic of more highly polymerized molecules to those characteristic of relatively less polymerized molecules as a result of the breakdown of polymerized procyanidins during the treatment. These phenomena have 
already been documented in our previous report (Hamauzu et al, 2007), but that experiment was conducted with $5 \%$ of citric acid. It is well known that procyanidins are transformed to cyanidin when broken down by heating with mineral acids (Porter, Hrstich \& Chan, 1986; Santos-Buelga \& Scalbert, 2000). However, it also has been shown that, even with $\mathrm{HCl}$, epicatechin monomers and dimers can be produced from oligomers if experimental conditions are $\mathrm{pH} 2.0$ and $37^{\circ} \mathrm{C}$ (Spencer, Chaudry, Pannala, Srai, Debnam \& Rice-Evans, 2000). In the current experiment with citric acid, the conditions seemed to be moderate, so that the procyanidins were partially transformed to cyanidin and their breakdown to (-)-epicatechin and dimer B2 occurred. According to Spencer et al. (2000), the procyanidin dimer seems to be more stable than other oligomers and this is supported by our observation that the (-)-epicatechin and procyanidin B2 levels increased during heat treatment.

Protocatechuic acid was detected clearly in the samples subjected to long time heating (CPT-12), indicating that the heat treatment decomposed polyphenols not only to the levels of monomers but also to smaller molecules such as phenolic acids. It has been known that protocatechuic acid result from thermal processing of flavonols (Buchner, Krumbein, Rohn \& Kroh, 2006) or from cyanidin (Sadilova, Stintzing \& Carle, 2006). Sadilova et al. (2006) reported that protocatechuic acid concentration was found to increase in purified anthocyanin isolates from some plant materials after heat exposure at $95{ }^{\circ} \mathrm{C}$ and $\mathrm{pH}$ 1. In current experiment, protocatechuic acid observed especially in a long-heated sample may be a result of cleavage of cyanidin that derived from 'extention units' of procyanidins.

The clump of polymeric procyanidins partially decreased during $2 \mathrm{~h}$ of heat treatment but it increased as denatured forms thereafter with increasing heating time. Because the absorbance increased in the visible light range, elongation of conjugated diene seems to have occurred. In addition, new components appeared with an 
absorption maximum of $458 \mathrm{~nm}$ after heat treatment. From the shape of their absorption spectra, it is likely that those components have a xanthylium structure. It has been reported that procyanidins or catechins produce xanthylium pigments by polymerization in the presence of an organic acid (Labrouche, Clark, Prenzler \& Scollary, 2005; Jurd \& Somers, 1970).

The increase in the antioxidant properties of Chinese quince polyphenols during heat treatment seemed to be due to changes in their composition and proportions, such as would occur through the lowering of the degree of polymerization and re-polymerization to form new components. The antioxidant properties of oligomeric procyanidins have been reported to be superior to those of polymers (Lu \& Foo, 2000).

\subsection{In Vivo Antioxidant Activity and Aromatic Acids in Rat Plasma}

The antioxidant activity of rat plasma as assessed by the FRAP method tended to increase after oral administration of $\mathrm{CP}$, and the tendency was stronger in rats given $\mathrm{CP}$ with citric acid (CPT-0) (Figure 3). The FRAP was also higher in rats given CPT-2 or CPT-8 than in the plasma of the controls, and in rats given CPT-2, the increase was significant $(P<0.05)$.

Aromatic acids detected in rat plasma $2 \mathrm{~h}$ after the administration of Chinese quince polyphenols were 3-hydroxyphenylacetic acid, protocatechuic acid, 4-hydroxybenzoic acid, vanillic acid, and hippuric acid (Figure 4). An additional peak $(\mathrm{m} / \mathrm{z}$ 209, peak 7) was also detected, indicating a possible metabolite that may have been 3,4-dihydroxyvaleric acid. However, we could not confirm this using its product ions. Although these aromatic acids were also detected in the controls' plasma, protocatechuic acid increased in the plasma of all rats after the administration of polyphenols (Figure 5). Moreover, vanillic acid levels increased significantly in the plasma of rats administered CPT-2 and CPT-8, being $4.7 \mu \mathrm{M}$ and $3.1 \mu \mathrm{M}$, respectively 
(4.7 and 3.1 times, respectively, that of the control or CP group $(1.0 \mu \mathrm{M})$ ). Because it has been reported that vanillic acid is transformed from protocatechuic acid by catechol- $O$-methyl transferase (COMT) in the liver (Gonthier et al., 2003b), the total amount of those two compounds (PCA+VA) was also compared in Figure 5. The concentration of PCA+VA in the CPT-2 group $(5.8 \mu \mathrm{M})$ was significantly higher $(P<$ 0.05) than that of other groups and it was approx. 4 times, 2.5 times and 2 times that of the control, CP and CPT-0 groups, respectively. Levels of hippuric acid tended to be higher in rats administered CPT than those of the control or CP group. However, they did not seem to reflect the administration of polyphenols; rather they seemed to be related to the citric acid administered with polyphenols. The plasma levels of two other aromatic acids, 3-hydroxyphenylacetic acid and 4-hydroxybenzoic acid, did not show any particular trends, with a concentration of $7.0-12 \mu \mathrm{M}$ in the former and $60-82 \mu \mathrm{M}$ in the latter.

These results suggested that polyphenols from Chinese quince fruit might raise the antioxidant activity of plasma after intake, and that heating the polyphenols in the presence of an organic acid could improve the effect by increasing the plasma concentrations of vanillic acid. Administration of CPT-2 resulted in the most effective increase in the FRAP, which might be explained by the fact that CPT-2 had the lowest proportion of polymeric components and the highest proportion of monomers, dimers and oligomers. It has been thought that procyanidins that are larger than trimers are unlikely to be absorbed in the small intestine in their native forms (Manach, Scalbert, Morand, Rémésy, Jiménez, 2004) and that, therefore, polymeric procyanidins may not contribute to an increase in the antioxidant activity of plasma in their intact forms. However, they can be absorbed once they reach the colon and are subjected to metabolism by the microflora there into various aromatic acids (Santos-Buelga \& Scalbert, 2000; Déprez, Brezillon, Rabot, Philippe, Mila, Lapierre et al., 2000; Gonthier, 
Cheynier, Donovan, Manach, Morand, Mila, 2003a), and act as functional compounds. The yields of aromatic acids of microbial origin have been reported to decrease with an increase in the degree of polymerization of procyanidins (Gonthier, Donovan, Texier, Felgines, Rémésy, Scalbert, 2003b). Therefore, breakdown of procyanidins into smaller polymers after $2 \mathrm{~h}$ of heating in the presence of $2 \%$ citric acid seemed to be an effective method of bringing about a high yield of aromatic acids by the acceleration of microbial metabolism. The highest concentration of vanillic acid was detected in the plasma of rats that had received CPT-2, indicating that metabolism of the polyphenols into aromatic acids and the intestinal absorption of those metabolites were most active in rats given CPT-2.

Because protocatechuic acid was detected in Chinese quince polyphenols especially in long time heated samples, this compound detected in rat plasma seems to be partly result of direct absorption from the samples administered. However, plasma concentration of protocatechuic acid (and vanillic acid, the metabolite of protocatechuic acid) did not reflect the tendency of the efficacy of heating time. Therefore, the action of intestinal flora of rats also might be important to increase of plasma concentration of these phenolic acids. 3-Hydroxyphenylacetic acid, protocatechuic acid and 4-hydroxybenzoic acid have been reported to be produced from the metabolism of (+)-catechin, (-)-epicatechin or procyanidins by the intestinal microflora, whereas vanillic acid might have been transformed from protocatechuic acid by COMT in the liver (Gonthier et al., 2003b). Among these aromatic metabolites, only levels of protocatechuic and vanillic acids reflected the effect of administration of $\mathrm{CP}$ or heat-treated CP; the other aromatic acids might have been derived from other ingested items such as pre-fasting food components or the citric acid administered with the polyphenols. Protocatechuic acid increased in the plasma of all rats given Chinese quince polyphenols and vanillic acid increased in the plasma of rats given CPT-2 and 
CPT-8, reflecting a positive effect of heat treatment. Since vanillic acid exists as protocatechuic acid before transformation by COMT in the liver, the total concentration of protocatechuic acid and vanillic acid seemed to reflect the total amount of the incorporated protocatechuic acid. This incorporation may have occurred in two ways: 1) direct incorporation from breakdown products of Chinese quince polyphenols; 2) indirect incorporation after that heated polyphenols were subjected to decompose by intestinal microflora. Since heat treatment must have advantage for both type of incorporation, these two compounds in the plasma could be useful as indicators for the intake of Chinese quince polyphenols and especially for the effect of the heat treatment on them.

It has been reported that protocatechuic acid and vanillic acid have antioxidant activity (Mansouri, Makris \& Kefalas, 2005; Guan, Bao \& Jiang, 2006; Shyamala, Naidu, Sulochanamma \& Srinivas, 2007). Therefore, these compounds might contribute to an increase in the FRAP of rats given Chinese quince polyphenols. In fact, the difference of the FRAP between the groups seemed to reflect the plasma PCA+VA level of them, although the actual FRAP value cannot be explained just from the concentration of aromatic acids detected.

Not only increased antioxidant activity but also other beneficial properties have been reported for those two aromatic acids. Protocatechuic acid has been reported to have an inhibitory effect on histamine release from rat mast cells (Osawa et al., 1999), anti-inflammatory activity (Fernández, Sáenz \& García, 1998) and an anti-cancer effect on TPA-induced tumor promotion (Tseng, Hsu, Lo, Chu, Chou, Huang et al., 1998). In addition, recent reports have shown that protocatechuic acid could inhibit the formation of $\mathrm{H}_{2} \mathrm{O}_{2}$-induced reactive oxygen species at the cytosolic level and also could inhibit $\mathrm{H}_{2} \mathrm{O}_{2}$-induced apoptotic events, such as the loss of mitochondrial functioning and DNA fragmentation (Tarozzi, Morroni, Hrelia, Angeloni, Marchesi, Cantelli-Forti et al., 
2007). Vanillic acid has been reported to have antimicrobial activity (De Moura, Pereira, Januário, França, Dias, 2004), inhibitory effects on platelet aggregation (Yasuda, Takasawa, Nakazawa, Ueda \& Ohsawa, 2003) and a stimulatory effect on the expression of human endothelial NO synthase (Wallerath, Li, Gödtel-Ambrust, Schwarz \& Förstermann, 2005). Therefore, a heat treatment-induced increase in the plasma concentration of these aromatic acids may confer health benefits through their effects on the circulatory system.

There are some additional advantages to the breakdown of Chinese quince polyphenols by heat treatment in the presence of citric acids. The treatment can increase in their bioavailability, as indicated by the observed increase in monomers such as (-)-epicatechin (which can be absorbed in their intact forms) (Manach et al., 2004). In the current experiment, however, this compound could not be detected in any of the rat plasma samples. The proportion of monomers in the polyphenolic samples given to rats was too small for monomers to be detected in the plasma after administration, even CPT-2 (it contained only 3\% of total polyphenols). Moreover, using citric acid might have an additional effect on the intestinal absorption of polyphenols. The FRAP and PCA+VA concentration were both higher in the plasma of rats given CPT-0 than in the rats given $\mathrm{CP}$, although the difference was not significant, suggesting that the presence of citric acid might affect the absorption of polyphenols. Yamashita et al. (Yamashita, Sakane, Harada, Sugiura, Koda, Kiso et al., 2002) reported that tartaric acid enhanced the absorption of catechin in rats. Another beneficial aspect of the breakdown of Chinese quince polyphenols by heat treatment is a decreased amount of highly polymerized procyanidins. Highly polymerized procyanidins are the main component of Chinese quince polyphenols, and by having strong tannic activity, they are associated with some food problems such as astringency and may be related to some types of iron deficiency (Santos-Buelga \& Scalbert, 2000). Thus, the heat-induced breakdown of 
polymeric procyanidins is beneficial because of decrease in astringency and increase in functionality although polymeric procyanidins themselves also may have some beneficial functions such as antioxidant activity, antiulcerative activity, anti-influenza viral activity (Hamauzu et al., 2005, 2006) and anti-proliferative activity (Miura et al., 2008). Polymeric procyanidins may have important functions in the digestive tract rather than in the circulatory system.

In conclusion, the breakdown of Chinese quince polyphenols by heating in the presence of citric acid seems to be an effective method to improve their biological activity through the increasing of lower molecular compounds and the in vivo production of aromatic acids such as protocatechuic and vanillic acids. The treatment could lower the proportion of polymeric procyanidins and increase the proportion of smaller molecules, such as dimers, monomers and protocatechuic acid. Thus, their ability to be absorbed in the small intestine and susceptibility to metabolism by microflora increases and the plasma concentration of aromatic acids of microbial origin will also increase. However, the results also suggest that heating for too long a period may decrease the efficiency of the treatment. The appropriate conditions to improve the health-promoting properties of Chinese quince polyphenols are under investigation.

\section{References}

Benzie, I. F. \& Strain, J. J. (1996). The ferric reducing ability of plasma (FRAP) as a measure of "antioxidant power": The FRAP assay. Analytical Biochemistry, 239, $70-76$.

Buchner, N., Krumbein, A., Rohn, S. \& Kroh, L. W. (2006) Effect of thermal processing on the flavonols rutin and quercetin. Rapid Communications in Mass Spectrometry, 20, 3229-3235. 
De Moura, R. M. X., Pereira, P. S., Januário, A. H., França, S. C. \& Dias, D. A. (2004). Antimicrobial screening and quantitative determination of benzoic acid derivative of Gomphrena celosioides by TLC-densitometry. Chemical \& Pharmaceutical Bulletin, 52, 1342-1344.

Déprez, S., Brezillon, C., Rabot, S., Philippe, C., Mila, I., Lapierre, C. \& Scalbert, A. (2000). Polymeric proanthocyanidins are catabolized by human colonic microflora into low-molecular-weight phenolic acids. Journal of Nutrition, 130, 2733-2738.

Fernández, M. A., Sáenz, M. T., García, M. D. (1998). Anti-inflammatory activity in rats and mice of phenolic acids isolated from Scrophularia frutescens. Journal of Pharmacy and Pharmacology, 50, 1183-1186.

Gonthier, M.-P., Cheynier, V., Donovan, J., Manach, C., Morand, C., Mila, I., Lapierre, C. \& Rémésy, C. (2003a). Microbial aromatic acid metabolites formed in the gut account for a major fraction of the polyphenols excreted in urine of rats fed wine polyphenols. Journal of Nutrition, 133, 461-467.

Gonthier, M.-P., Donovan, J. L., Texier, O., Felgines, C., Rémésy, C. \& Scalbert, A. (2003b). Metabolism of dietary procyanidins in rats. Free Radical Biology and Medicine, 837-844.

Guan, S., Bao, Y.-M., Jiang, B. \& An, L.-J. (2006). Protective effect of protocatechuic acid from Alpinia oxyphylla on hydrogen peroxide-induced oxidative PC12 cell death. Europian Journal of Pharmacology, 538, 73-79.

Hamauzu, Y., Yasui, H., Inno, T., Kume, C. \& Omanyuda, M. (2005). Phenolic profile, antioxidant property, and anti-influenza viral activity of Chinese quince (Pseudocydonia sinensis Schneid.), quince (Cydonia oblonga Mill.), and apple 
(Malus domestica Mill.) fruits. Journal of Agricultural and Food Chemistry, 53, 928-934.

Hamauzu, Y., Inno, T., Kume, C., Irie, M. \& Hiramatsu, K. (2006). Antioxidant and antiulcerative properties of phenolics from Chinese quince, quince, and apple fruits. Journal of Agricultural and Food Chemistry, 54, 765-772.

Hamauzu, Y., Kume, C., Yasui, H. \& Fujita, T. (2007). Reddish coloration of Chinese quince (Pseudocydonia sinensis) procyanidins during heat treatment and effect on antioxidant and antiinfluenza viral activities. Journal of Agricultural and Food Chemistry, 55, 1221-1226.

Jurd, L. \& Somers, T. C. (1970). The formation of xanthylium salts from proanthocyanidins. Phytochemistry, 9, 419-427.

Labrouche, F., Clark, A. C., Prenzler, P. D. \& Scollary, G. R. (2005). Isomeric influence on the oxidative coloration of phenolic compounds in a model white wine: comparison of (+)-catechin and (-)-epicatechin. Journal of Agricultural and Food Chemistry, 53, 9993-9998.

Lu, Y. \& Foo, L. Y. (2000). Antioxidant and radical scavenging activities of polyphenols from apple pomace. Food Chemistry, 68, 81-85.

Manach, C., Scalbert, A., Morand, C., Rémésy, C. \& Jiménez, L. (2004). Polyphenols: food sources and bioavailability. American Journal of Clinical Nutrition, 79, $727-747$.

Mansouri, A., Makris, D. P. \& Kefalas, P. (2005). Determination of hydrogen peroxide scavenging activity of cinnamic and benzoic acids employing a highly sensitive peroxyoxalate chemiluminescence-based assay: structure-activity relationships. Journal of Pharmaceutical and Biomedical Analysis, 39, 22-26. 
Miura, T., Chiba, M., Kasai, K., Nozaka, H., Nakamura, T., Shoji, T., Kanda, T., Ohtake, Y. \& Sato, T. (2008) Apple procyanidins induce tumor cell apoptosis through mitochondrial pathway activation of caspase-3. Carcinogenesis, 29,585-593.

Mohri, Y., Sagehashi, M., Yamada, T., Hattori, Y., Morimura, K., Kamo, T., Hirota, M. \& Makabe, H. (2007). An efficient synthesis of procyanidins. Rare earth metal Lewis acid catalyzed equimolar condensation of catechin and epicatechin. Tetrahedron Letters, 48, 5891-5894.

Oku, H., Ueda, Y. \& Ishiguro, K. (2003). Antipruritic effects of the fruits of Chaenomeles sinensis. Biological \& Pharmaceutical Bulletin, 26, 1031-1034.

Osawa, K., Miyazaki, K., Imai, H., Arakawa, T., Yasuda, H. \& Takeya, K. (1999). Inhibitory effects of Chinese quince (Chaenomeles sinensis) on hyaluronidase and histamine release from rat mast cells (in Japanese with English summary). Natural Medicines, 53, 188-193.

Porter, L. J., Hrstich, L. N. \& Chan, B. G. (1986). The conversion of procyanidins and prodelphinidins to cyanidin and delphinidin. Phytochemistry, 25, 223-230.

Sadilova, E., Stintzing, F. C. \& Carle, R. (2006) Thermal degradation of acylated and nonacylated anthocyanins. Journal of Food Science, 71, 504-512.

Santos-Buelga, C. \& Scalbert, A. (2000). Proanthocyanidins and tannin-like compounds - nature, occurrence, dietary intake and effects on nutrition and health. Journal of the Science of Food and Agriculture, 80, 1094-1117.

Shyamala, B. N., Naidu, M. M., Sulochanamma, G. \& Srinivas, P. (2007). Studies on the antioxidant activities of natural vanilla extract and its constituent compounds 
through in vitro models. Journal of Agricultural and Food Chemistry, 55, $7738-7743$.

Spencer, J. P. E., Chaudry, F., Pannala, A. S., Srai, S. K., Debnam, E. \& Rice-Evans, C. (2000). Decomposition of cocoa procyanidins in the gastric milieu. Biochemical and Biophysical Research Communications, 272, 236-241.

Tarozzi, A., Morroni, F., Hrelia, S., Angeloni, C., Marchesi, A., Cantelli-Forti, G. \& Hrelia, P. (2007). Neuroprotective effects of anthocyanins and their in vivo metabolites in SH-SY5Y cells. Neuroscience Letters, 424, 36-40.

Tseng, T., Hsu, J., Lo, M., Chu, C., Chou, F., Huang, C. \& Wang, C. (1998). Inhibitory effect of Hibiscus protocatechuic acid on tumor promotion in mouse skin. Cancer Letters, 126, 199-207.

Wallerath, T., Li, H., Gödtel-Ambrust, U., Schwarz, P. M. \& Förstermann, U. (2005). A blend of polyphenolic compounds explains the stimulatory effect of red wine on human endothelial NO synthase. Nitric Oxide, 12, 97-104.

Yamashita, S., Sakane, T., Harada, M., Sugiura, N., Koda, H., Kiso, Y. \& Sezaki, H. (2002). Absorption and metabolism of antioxidative polyphenolic compounds in red wine. Annals of the New York Academy of Sciences, 957, 325-328.

Yasuda, T., Takasawa, A., Nakazawa, T., Ueda, J. \& Ohsawa, K. (2003). Inhibitory effects of urinary metabolites on platelet aggregation after orally administering Shimotsu-To, a traditional Chinese medicine, to rats. Journal of Pharmaceutical and Pharmacology, 55, 239-244. 


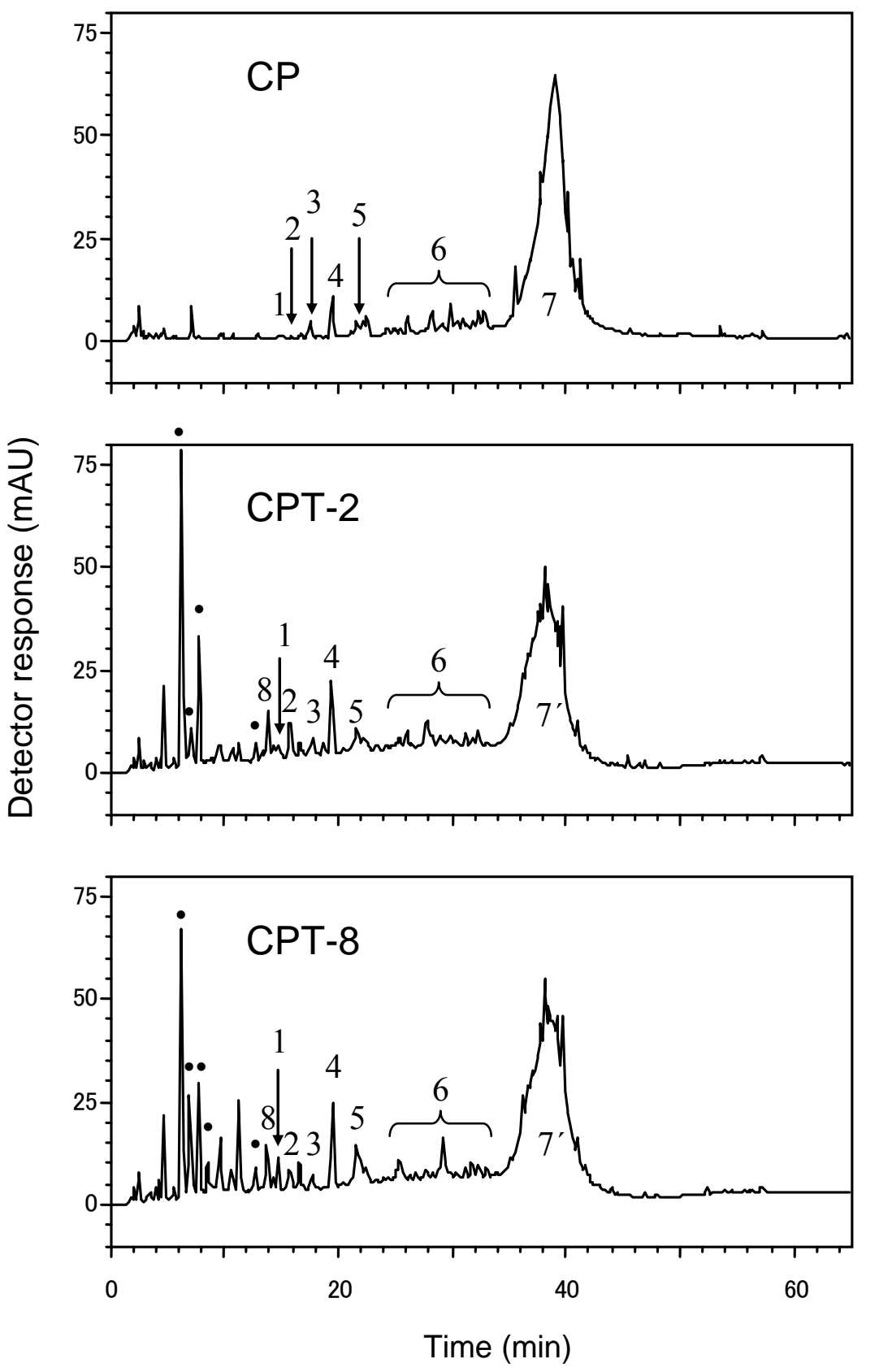

Figure 1. RP-HPLC profiles of Chinese quince phenolics (CP) and $\mathrm{CP}$ subjected to heating for $2 \mathrm{~h}(\mathrm{CPT}-2)$ and $8 \mathrm{~h}$ (CPT- 8$)$ in the presence of citric acid. 1, procyanidin B3; 2, (+)-catechin; 3, procyanidin $\mathrm{B} 4 ; 4$, procyanidin $\mathrm{B} 2 ; \mathrm{5}$, (-)-epicatechin; 6, oligomeric procyanidins; 7, clump of polymeric procyanidins; $7^{\prime}$, clump of polymeric components including procyanidin polymers and denatured polyphenols. Peaks indicated with '•' are new peaks with absorption spectra were similar to that of catechin. 

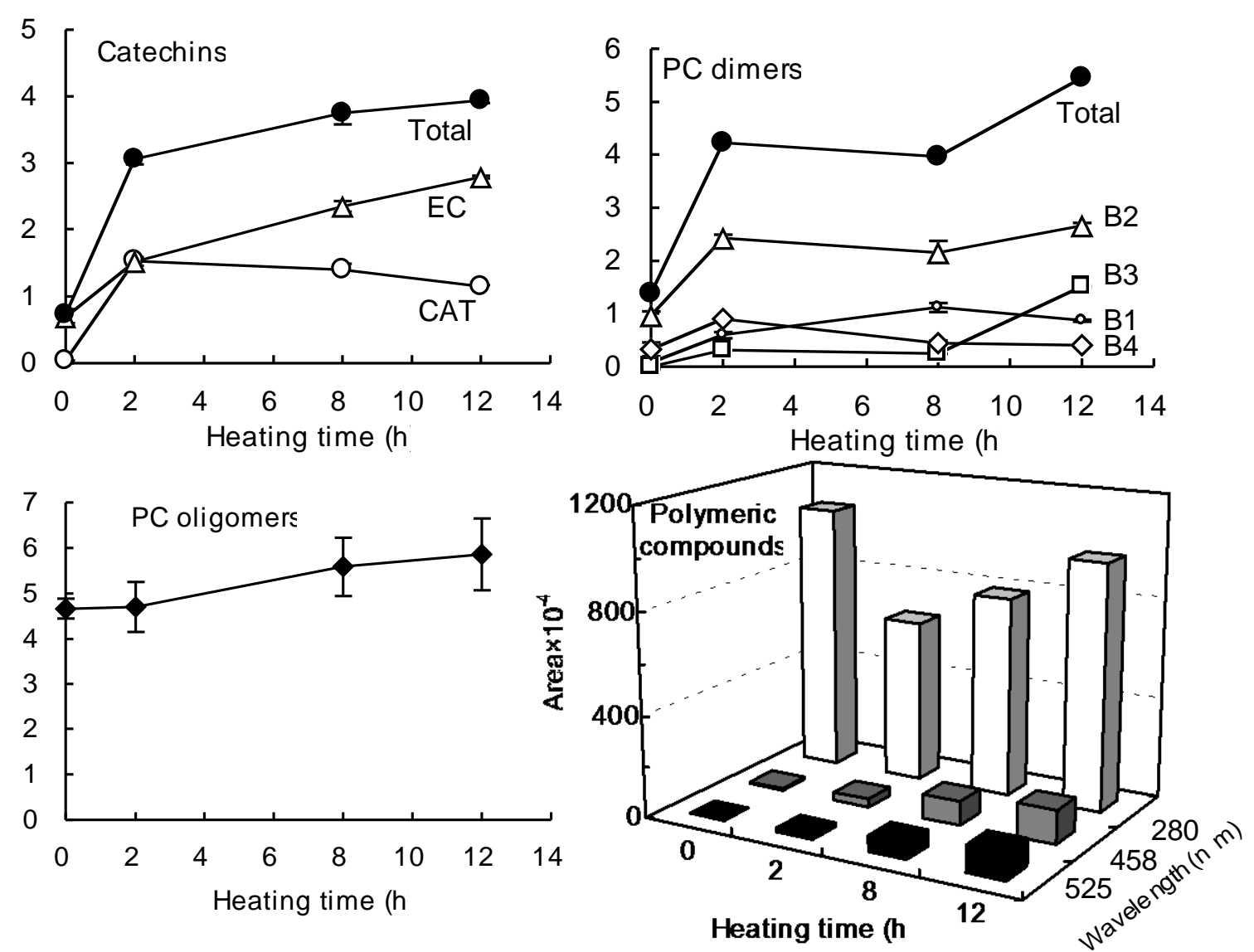

Figure 2. Change in catechin and procyanidin concentrations and the area of polymeric compounds detected at 280, 458 and $525 \mathrm{~nm}$ during the heating of the Chinese quince phenolic solution in the presence of citric acid. Bars indicate SE $(n=3)$. EC, (-)-epicatechin; CAT, (+)-catechin; PC, procyanidin; B1-B4, procyanidin B1-B4 


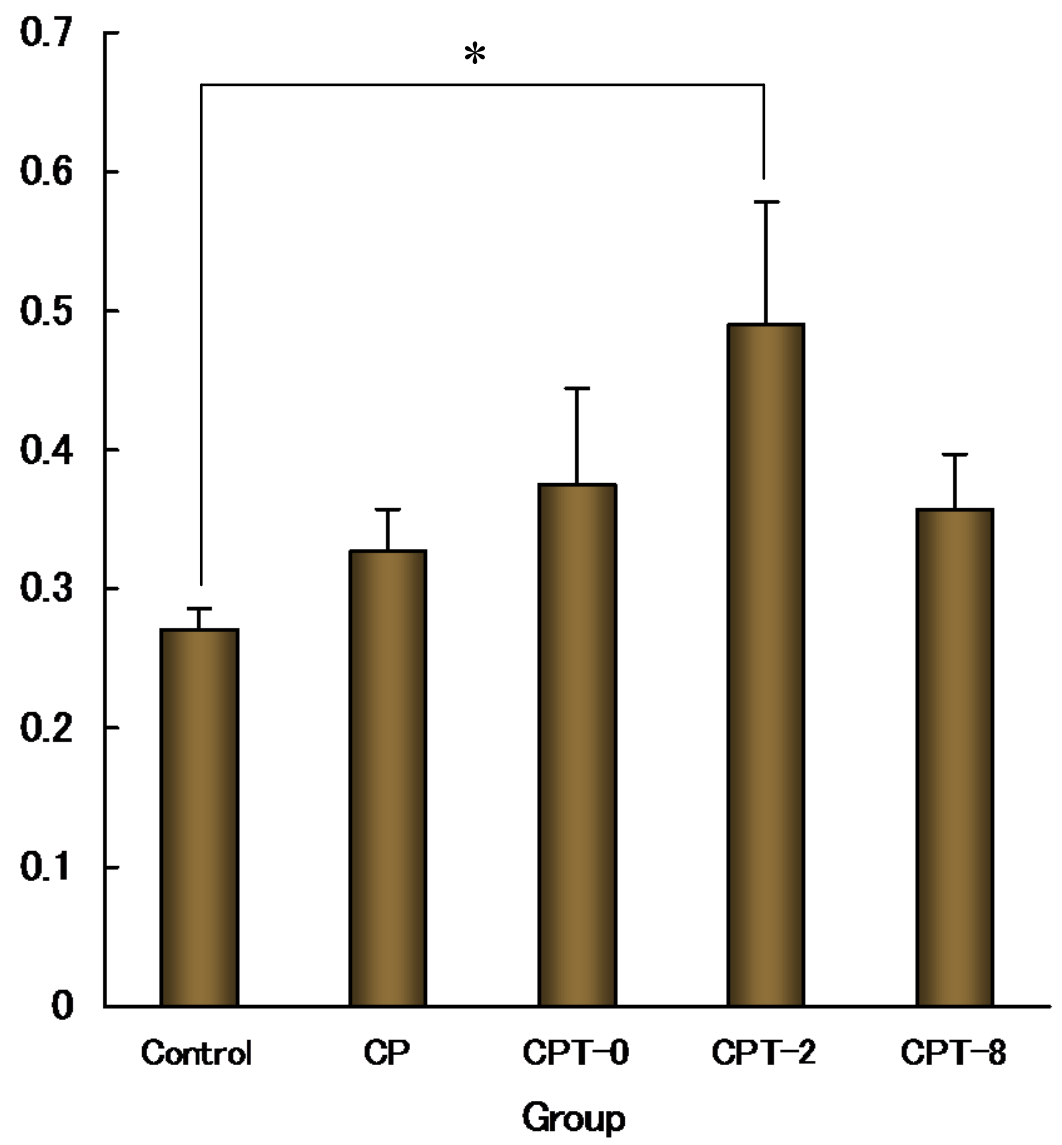

Figure 3. Ferric reducing ability of the plasma (FRAP) of control rats and rats administered Chinese quince phenolics, some of which were subjected to heat treatment (see text for explanation of group codes). Bars indicate SE ( $n=10$ for control and CP group; $n=4-5$ for CPT group). $* P<0.05$ (by Tukey-Kramer test) 


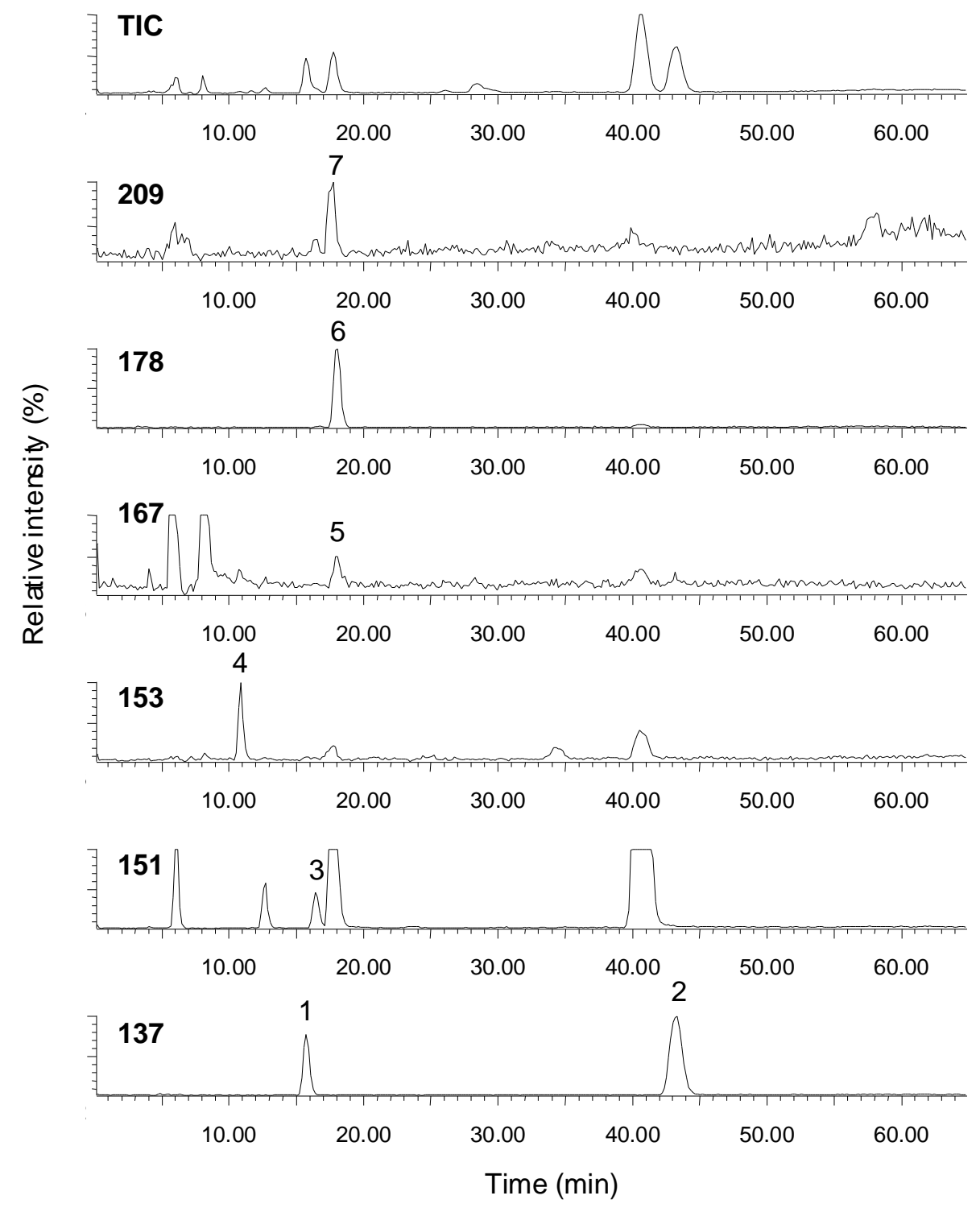

Figure 4. LC/MS single ion recording (SIR) mass chromatogram profile of representative rat plasma after the administration of Chinese quince phenolics.

Peaks: 1 = 4-hydroxybenzoic acid; $2=$ salicylic acid (IS); $3=$ 3-hydroxyphenylacetic acid; $4=$ protocatechuic acid; $5=$ vanillic acid; $6=$ hippuric acid; 7 = 3,4-dihydroxyphenylvaleric acid (?). 

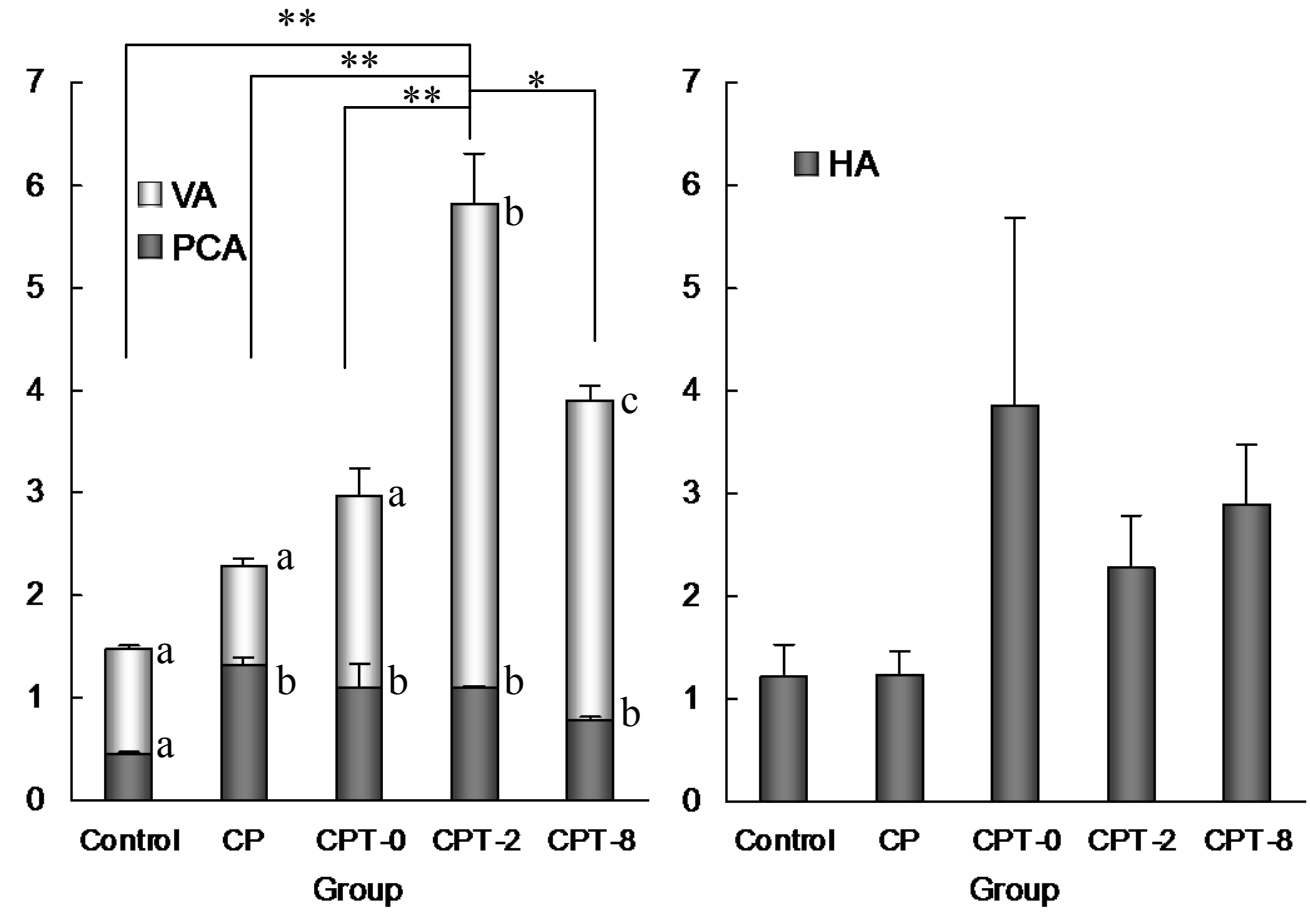

Figure 5. Aromatic acid concentrations in the plasma of control and trial rats $2 \mathrm{~h}$ after the administration of Chinese quince phenolics, some of which were subjected to heat treatment (see text for explanation of group codes). PCA, protocatechuic acid; VA, vanillic acid; HA, hippuric acid.

Bars indicate SE $(n=4-5)$. $* P<0.05, * * P<0.01$ (by one-way ANOVA and Tukey-Kramer test for total amount of PCA and VA) For each administration, different letters in PCA or VA were significantly different $(P<0.05)$. 